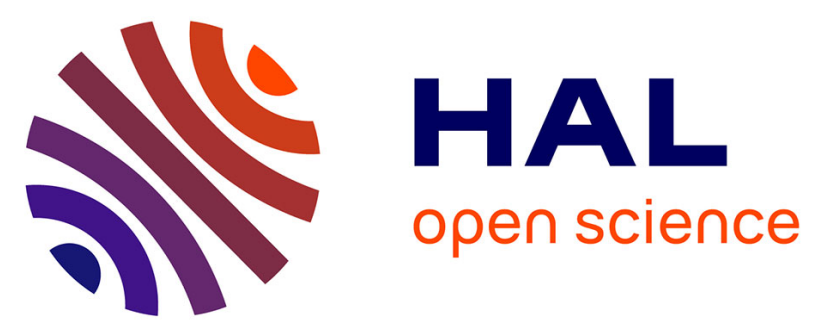

\title{
Automatic laser alignment for multifocal microscopy using a LCOS-SLM and a 32x32 pixel CMOS SPAD array
}

David Tyndall, Richard Walker, Krzysztof Nguyen, Rémi Galland, Jie Gao, Irène Wang, Meike Kloster, Antoine Delon, Robert Henderson

\section{To cite this version:}

David Tyndall, Richard Walker, Krzysztof Nguyen, Rémi Galland, Jie Gao, et al.. Automatic laser alignment for multifocal microscopy using a LCOS-SLM and a 32x32 pixel CMOS SPAD array. Proceedings of SPIE, the International Society for Optical Engineering, 2011, 8086, pp.80860S. 10.1117/12.889738 . hal-00654724

\section{HAL Id: hal-00654724 https://hal.science/hal-00654724}

Submitted on 22 Dec 2011

HAL is a multi-disciplinary open access archive for the deposit and dissemination of scientific research documents, whether they are published or not. The documents may come from teaching and research institutions in France or abroad, or from public or private research centers.
L'archive ouverte pluridisciplinaire HAL, est destinée au dépôt et à la diffusion de documents scientifiques de niveau recherche, publiés ou non, émanant des établissements d'enseignement et de recherche français ou étrangers, des laboratoires publics ou privés. 


\title{
Automatic laser alignment for multifocal microscopy using a LCOS SLM and a $32 \times 32$ pixel CMOS SPAD array
}

\author{
David Tyndall ${ }^{*}$, Richard Walker ${ }^{a}$, Krzysztof Nguyen ${ }^{a}$, Rémi Galland ${ }^{b}$, Jie Gao $^{b}$, Irène Wang ${ }^{b}$, \\ Meike Kloster ${ }^{b}$, Antoine Delon ${ }^{b}$ and Robert Henderson ${ }^{a}$ \\ ${ }^{a}$ Integrated Micro and Nano Systems, The University of Edinburgh, Edinburgh, EH9 3JL, UK; \\ ${ }^{b}$ Laboratoire Interdisciplinaire de Physique UMR 5588, Université de Grenoble 1 / CNRS, BP 87, \\ 38402, Saint Martin d'Hères, France
}

\begin{abstract}
Alignment of a laser to a point source detector for confocal microscopy can be a time-consuming task. The problem is further exacerbated when multiple laser excitation spots are used in conjunction with a multiple pixel single photon detector; in addition to $\mathrm{X}, \mathrm{Y}$ and $\mathrm{Z}$ positioning, pixels in a $2 \mathrm{D}$ array detector can also be misaligned in roll, pitch and yaw with respect to each other, causing magnification, rotation and focus variation across the array. We present a technique for automated multiple point laser alignment to overcome these issues using closed-loop feedback between a laser illuminated computer controlled Liquid Crystal on Silicon Spatial Light Modulator (LCOS-SLM) acting as the excitation source and a $32 \times 32$ pixel CMOS Single Photon Avalanche Diode (SPAD) array as the multiple pixel detection element. The alignment procedure is discussed and simulated to prove its feasibility before being implemented and tested in a practical optical system. We show that it is possible to align each independent laser point in a sub-second time scale, significantly simplifying and speeding up experimental set-up times. The approach provides a solution to the difficulties associated with multiple point confocal laser alignment to multiple point detector arrays, paving the way for further advances in applications such as Fluorescence Correlation Spectroscopy (FCS) and Fluorescence Lifetime Imaging Microscopy (FLIM).
\end{abstract}

Keywords: Confocal, SLM, SPAD, FCS, FLIM, TCSPC, photon counting

\section{INTRODUCTION}

Fluorescence Correlation Spectroscopy (FCS) and Fluorescence Lifetime Imaging Microscopy (FLIM) both benefit greatly from advanced confocal microscopy techniques such as confocal laser scanning and spinning disk microscopy, providing improved resolution, depth selectivity and photon efficiency over widefield techniques. Although theses techniques are well practised, producing high throughput imaging of multiple positions in a sample, the imaging points are still captured sequentially and do not provide fully parallel simultaneous data acquisitions. Applications in living cells would greatly benefit from the possibility of performing truly simultaneous measurements at multiple locations. Not only would this provide more complete information on the cellular machinery, but also, thanks to the parallel acquisitions, one would obtain statistically significant results in a more time-efficient manner.

There has been recent interest in performing multiple point confocal microscopy for fluorescence experiments using microlens arrays, diffractive optical elements (DOE) ${ }^{1}$ and spatial light modulators (SLM). ${ }^{2,3}$ However, there are a number of drawbacks to using microlenses and DOEs for multifocal microscopy; they are very difficult to align to small (pinhole) detection points as manual micrometer adjustments are required, the fan out must ideally be matched to the detector resolution and their static nature means that turning beams on and off independently is not possible. There is much more flexibility offered by SLMs; they can be aligned using computer control and any number of beams (limited by available laser power) can be created in any user-defined pattern. Although SLMs can be controlled by software, to the best of our knowledge only partially automated alignment procedures have been introduced. ${ }^{3}$ With the recent introduction of arrays of spatially separated pinhole-like single photon detector arrays, capable of high speed photon counting and time correlated imaging on a per-pixel basis, ${ }^{4}$ an enhanced approach to multifocal alignment is required. We present a completely automated procedure for accurately aligning multiple laser spots with detectors in a 2D array.

*E-mail: d.tyndall@ed.ac.uk, Telephone: +44 (0)131 650 5658; Website: www.css.eng.ed.ac.uk 


\section{MATERIALS AND METHODS}

\subsection{Optical Set-up}

All measurements were performed on a homebuilt experimental set-up, based on an inverted microscope body (Olympus IX70). A diagram of the optical set-up can be seen in Figure 1.

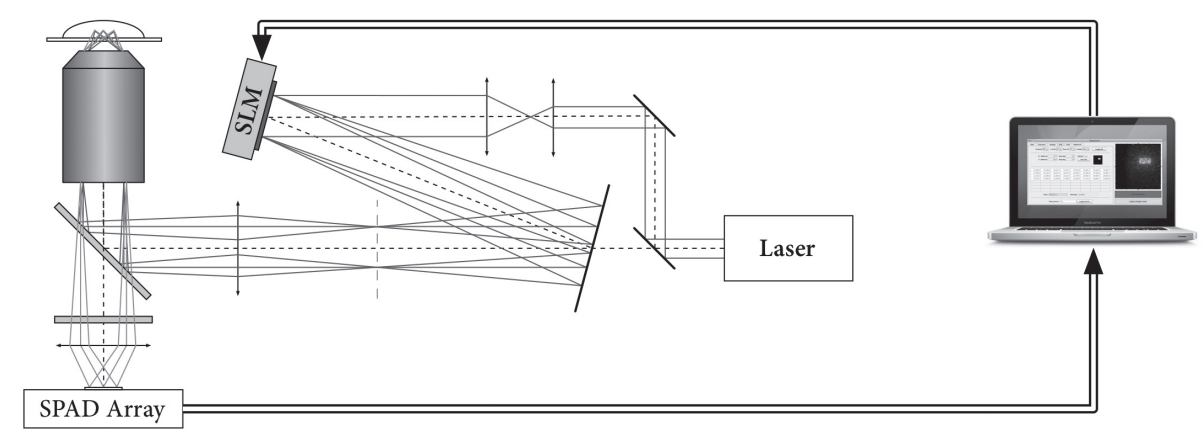

Figure 1: Closed-loop feedback system and optical set-up with SLM and detector both being controlled by software on a PC.

Fluorescence excitation is performed using a solid-state laser emitting $20 \mathrm{~mW}$ of $488 \mathrm{~nm}$-wavelength continuous-wave light (85-BCD-020, Melles Griot). The beam is sent into a single-mode fibre in order to spatially clean its wavefront. It is then directed to the SLM, composed of $800 \times 60020 \mu \mathrm{m}$-pixels (LCOS-SLM X10468-01, Hamamatsu). Before reaching the SLM, the beam is expanded using a telescope $(\times 3)$ to cover its active area, and passed through a half-wave plate and a polariser (since this SLM only diffracts horizontally polarised radiation). When the adequate phase map is addressed to the SLM, the beam is focused in a plane which is optically conjugate with the microscope object plane. The beam is then collimated before being reflected by a dichroic mirror (PB 505, Olympus) toward a water immersion objective lens $(\times 60$, $\mathrm{NA}=1.2$, Olympus). This collimation lens is also used to image the SLM onto the back aperture of the objective lens to ensure that all the diffracted light is coupled into the objective. The laser power, measured at the exit of the fibre, is varied around $10 \mu \mathrm{W}$ for alignment and up to $1 \mathrm{~mW}$ for multiple spot creation (according to the total number of spots).

Fluorescence emitted by the sample is spectrally filtered (PH 510, Olympus) then directed to a side port of the microscope where it is sent towards the SPAD detector, a sub-array of which can be seen in Figure 2. Here, we use a pair of achromatic lenses, to produce a total magnification of $\times 12$ of the entire microscope. In this case, each SPAD active area acts as an individual pinhole for parallel confocal detection.

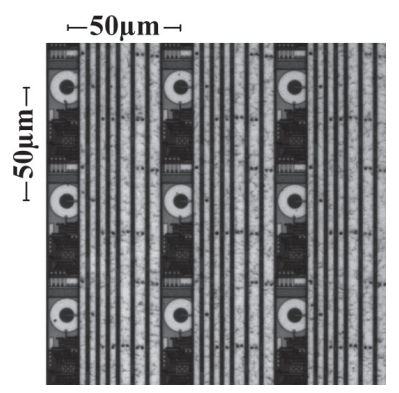

Figure 2: Image of a $3 \times 3$ sub-array $(150 \mu \mathrm{m} \times 150 \mu \mathrm{m})$ of the CMOS SPAD detector, showing the spatially separated detectors, associated timing and counting circuitry plus metal routing.

\subsection{SLM Laser Spot Generation}

To create an arbitrary set of diffraction limited spots in the microscope object plane, we display the corresponding phase map computed by a spherical wave and superposition approach on the SLM. ${ }^{5}$ We want the SLM to focus the incoming plane wave into a number of freely chosen spots at a finite distance. The principle of the algorithm is as described; we 
assume that each desired spot results from a converging spherical wave, then the phase function is obtained from the back-propagation and superposition of the spherical wavefronts in the plane of the SLM.

We define $r_{m}(x, y)$ as the distance between the pixel of the SLM at coordinates $x, y$ and the $m^{\text {th }}$ spot:

$$
r_{m}(x, y)=\sqrt{\left(x-x_{m}\right)^{2}+\left(y-y_{m}\right)^{2}+f^{2}}
$$

Spots are initially created in the same plane on the z-axis, optically conjugate with the microscope object plane. This plane, which can be considered as the SLM focal plane, is at a distance $f$ (we used $f=364 \mathrm{~mm}$ initially, before optimising) from the SLM. To generate $M$ spots, we apply the following phase pattern to the SLM:

$$
\phi(x, y)=\arg \left(\sum_{m=1}^{M} a_{m} \frac{\exp \left[-i \frac{2 \pi}{\lambda} r_{m}(x, y)+\theta_{m}\right]}{r_{m}(x, y)}\right)
$$

where $a_{m}$ are amplitude factors, that take into account the obliquity factor $\cos \psi_{m}(x, y)=f / r_{m}(x, y)$ and $\theta_{m}$ are random phases added as a method of dithering in order to reduce unwanted interferences.

This spherical wave and superposition algorithm has the advantage of relatively short computation times of approximately $200 \mathrm{~ms}$ per spot (on a modern $2.4 \mathrm{GHz}$ processor). Diffraction-limited spots can be generated with reasonably good efficiency, with the disadvantage that the laser power is not distributed uniformly between different spots. An iterative approach can be employed to improve the power distribution, with the drawback of much longer computation times.

\subsection{Detector Array}

The detector is a low dark count rate (DCR), $32 \times 32$ pixel Single Photon Avalanche Diode (SPAD) array implemented in a $130 \mathrm{~nm}$ CMOS imaging process with in-pixel circuitry for photon counting and time correlated imaging. ${ }^{4}$ The detector operates at a frame rate of $500 \mathrm{KHz}$ and each frame is capable of either counting up to 40 photons (limited by a SPAD dead-time of 50ns) or measuring a time correlated single photon counting (TCSPC) time stamp. Each SPAD has a circular active area of $6.7 \mu \mathrm{m}$ diameter, positioned with a pixel pitch of $50 \mu \mathrm{m}$ as can be seen in Figure 2. The small diameter active area acts as a virtual pinhole for confocal detection, but introduces difficulties in terms of laser alignment. The peak detection efficiency of the SPAD is $28 \%$ at a wavelength of $500 \mathrm{~nm}$, when biased $1.4 \mathrm{~V}$ above the breakdown of $14.4 \mathrm{~V}$. $^{6}$ The array has a DCR distribution with roughly $80 \%$ of pixels under $150 \mathrm{~Hz}$ but reaching up to $10 \mathrm{KHz}$ in the worst case. As only a small number of detectors are required for alignment, only sub- $150 \mathrm{~Hz}$ DCR SPADs are used for the reported results.

\subsection{Feedback System}

Both the detector and SLM are controlled using a custom self-contained Java application. The software is used to set up and acquire image data from the detector and compute and drive phase maps onto the SLM. It also acts as the feedback loop, altering the SLM phase map as necessary depending on the counts acquired from the imaging array. A system overview of all the components and the optical path can be seen in Figure 1.

\subsection{Sample preparation}

Alignment was performed using a $10 \mu \mathrm{M}$ solution of Sulforhodamine G (SRG) molecules (Radiant Dyes) in pure water, without further purification, to provide high count rates for a relatively low laser power. A droplet of this solution was deposited in a perfusion chamber on a microscope cover slide, to prevent evaporation during alignment.

\section{AUTOMATED ALIGNMENT PROCEDURE}

A naïve approach to alignment would consist of searching for the peak of the intensity profile by moving the calculated SLM phase map in X, Y and Z. Significant speed improvements can be gained if an estimate of the laser position in the detector plane could be made first. This position estimate would then be able to provide a good seed for a peak search, narrowing down the search area and speeding up the alignment process significantly. Without the estimation, the peak search could have to be carried out across a much larger distance (up to $70 \mu \mathrm{m}$ ) depending on the initial laser position.

Incorporating such a technique, the alignment procedure for multiple laser points will consist of the following steps; to begin with a single point is aligned using a position estimate followed by a peak search, then the detector geometry misalignments are calculated by using a peak search at specified detector positions, and finally from this information, multiple points can then be calculated without the need for further alignments. 


\subsection{Single Spot Position Estimate}

With prior knowledge of how a detected laser spot intensity drops off with distance from a single detector, one can use the intensities recorded at pixels surrounding a misaligned laser spot to estimate the position of that spot in X and Y. A procedure to achieve this is outlined below (assuming the centre of the SLM is within the perimeter of the detector array):

1: Calculate SLM phase map to create a laser spot at position $(0,0)$ in the SLM plane.

2: Capture a long exposure (hundreds of milliseconds) of photon count data from the full array. The laser spot will ensure that the four detectors surrounding it in a square, as shown in Figure 3, will contain the highest count rates.

3: Select the pixel with the highest count rate as the centre detector. In Figure 3, this will be the lower left pixel.

4: Search for the maximum-likelihood position of the four, $50 \mu \mathrm{m}$ spaced, intensity points in a look up table (LUT) of the known intensity profile.

5: Recalculate the SLM phase map position towards the calculated centre detector.

The performance of such an algorithm is heavily dependent on the count rates and the deviation of the intensity profile, as will be discussed in Section 4.

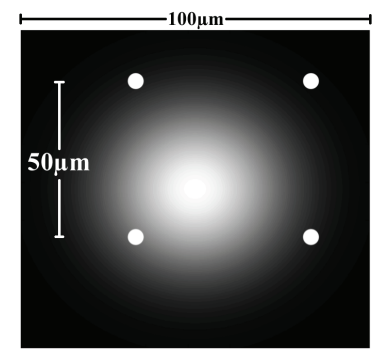

Figure 3: Ideal Gaussian intensity profile over a $100 \mu \mathrm{m}$ square with maximum intensity (white) down to a noise floor (black) showing a misaligned spot peak between four SPAD detectors (white circles).

\subsection{Single Spot Alignment (Peak Search)}

Once the position of a single spot has been estimated, fine-tuning in $\mathrm{X}, \mathrm{Y}$ and $\mathrm{Z}$ can then take place to find the peak of the intensity profile. A procedure for the peak search used is detailed below:

1: Capture count rate at an SLM position to one side in the X dimension (e.g. to the left)

2: if ( new count $>$ previous count )

3: $\quad$ Continue moving in this direction until ( new count $<$ previous count ), then break

4: else Capture count rate at an SLM position to the other side in the X dimension (e.g. to the right)

5: $\quad$ if ( new count $>$ previous count $)$

6: $\quad$ Continue moving in this direction until ( new count $<$ previous count ), then break

7: $\quad$ else Optimised, break

8: Repeat steps 1-7 for $\mathrm{Y}$, then $\mathrm{Z}$ dimensions

9: Repeat steps 1-8 until all dimensions are optimised (a maximum count has been reached)

Depending on the expected worse case distance this algorithm has to search, different values can be used for the searching step size. In the case of this work, all the alignments were performed using two iterations of this procedure with decreasing step sizes of $2 \mu \mathrm{m}$ and $0.2 \mu \mathrm{m}$.

\subsection{Multiple Point Alignment}

The procedure above is only necessary for aligning the first spot; since the SPAD array has a rigid geometry, additional spot positions can be estimated using the known pitch of the detector and finely adjusted to calculate for the detector misalignments. To do this, assume an ideally aligned array to calculate the position of spots to detectors perpendicular to the centre detector. Performing the peak search at these perpendicular detectors, one can calculate any non-idealities in magnification, focus and rotation of the entire array. The time taken to perform these searches will be dependent on how well or poorly aligned the detector and SLM are in relation to each other. The alignment of any additional laser spots can then be estimated using the gathered information, meaning only one position estimate and three accurate peak searches are required to align any number of laser spots within the field of view. 


\section{SIMULATION}

Simulations of the initial single point position estimation procedure were carried out in MATLAB assuming a Gaussian distributed, shot noise dominated intensity profile with a varying peak count, $C_{p e a k}$. The LUT is a 2D Gaussian with fixed $C_{\text {peak }}$. Figure 4 shows the ability of the initial position estimation part of the procedure (Section 3.1) to align within $1 \mu \mathrm{m}$ and the diameter $(6.7 \mu \mathrm{m})$ of the centre of the SPAD, with varying peak photon counts (4(a)) and standard deviation (4(b)).

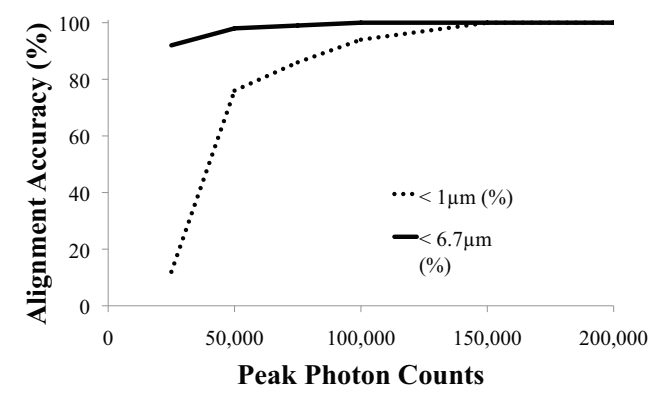

(a) Varying $C_{\text {peak }}$ with a fixed standard deviation of $36 \%$ of the pitch $(18 \mu \mathrm{m})$

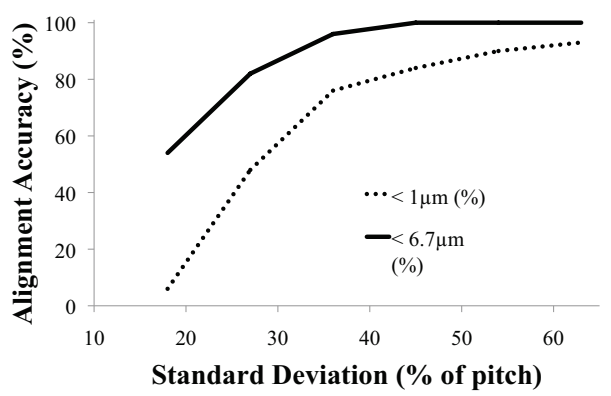

(b) Varying the standard deviation with fixed $C_{\text {peak }}$ of 100,000 counts.

Figure 4: Graphs showing the ability of the procedure to align within $1 \mu \mathrm{m}$ (dotted) and $6.7 \mu \mathrm{m}$ (solid - within active area) by varying $C_{\text {peak }}$ and standard deviation of the profile.

For the best alignment accuracy in the shortest time, a peak of 150,000 photon counts or greater is required, which is easily achieved with a bright alignment sample in under 1 second (in our case $10 \mu \mathrm{M}$ SRG provided a peak count rate of around $200 \mathrm{KHz}$ with under $10 \mu \mathrm{W}$ of laser power). As expected, alignment accuracy improves with larger standard deviation; our experimental results show that the standard deviation of $18 \mu \mathrm{m}(36 \%)$ is particularly well suited to the pixel pitch of the detector array, providing significant counts for alignment purposes but not providing a high optical cross-talk once accurately aligned (approximately $3 \%$ for neighbouring pixels). In practice, the intensity profile will not be a uniform 2D Gaussian, so the peak search is still essential to find the optimal position.

\section{RESULTS}

The algorithm is shown to be successfully capable of aligning multiple laser beams to multiple SPAD detectors. The alignment of a single point is verified by recalculating the SLM phase map to positions surrounding the aligned position and capturing count data. Examples of this are shown in Figures 5(a) to 5(c), where the centre pixel is the SLM position resulting from the alignment procedure. Figure 5(a) shows a relatively flat intensity profile approximating the SPAD active area, where a $\pm 1.5 \mu \mathrm{m}$ error provides $98 \% C_{\text {peak }}$. Figures 5(b) and 5(c) clearly show a non-ideal, elongated intensity profile causing slightly worse than simulated position estimates, but always improving the search area to within $10 \mu \mathrm{m}$ of the centre.

Given the spot calculation time of $200 \mathrm{~ms}$, exposures of $800 \mathrm{~ms}$ were taken for all SLM positions, providing a total searching step time of 1 second (this could be reduced with brighter samples/higher laser power). As expected, the initial single spot alignment took the longest to find the optimal position, taking 24 to 40 steps for our experiments. The perpendicular alignments could be optimised faster, taking 9 to 20 steps. A grid of $4 \times 8$ spots was then calculated using the misalignment information gathered from the three initial alignments, before each of the points was optimised to validate the accuracy of their calculation. The calculated centres of the 32 spots were found to be within $1.96 \mu \mathrm{m}(0.85 \mu \mathrm{m}$ average $)$ of the fully optimised centres, showing that further optimisation was not necessary. The alignment procedure was able to fully align any number of spots within the field of view in a fixed time of approximately 60 seconds, meaning over 60 spots could be calculated with a sub second average per spot. Figure 5(d) shows a CCD image of the 4x8 aligned laser spots. The SLM and SPAD array in this case were found to be misaligned in magnification (pitch=50.2 $\mu \mathrm{m})$ and rotation $\left(\theta=-2.6^{\circ}\right)$. 


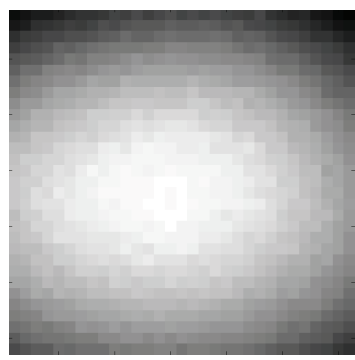

(a) $5 \times 5 \mu \mathrm{m}$ profile $(75 \mathrm{~K}-$ $100 \mathrm{~K}$ photon counts).

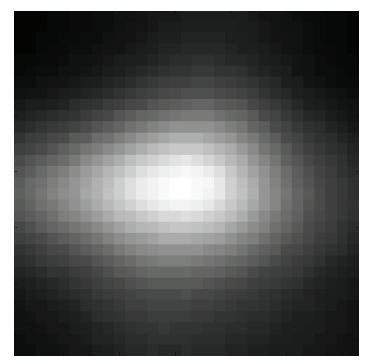

(b) $25 \times 25 \mu \mathrm{m}$ profile $(17 \mathrm{~K}$ $100 \mathrm{~K}$ photon counts).

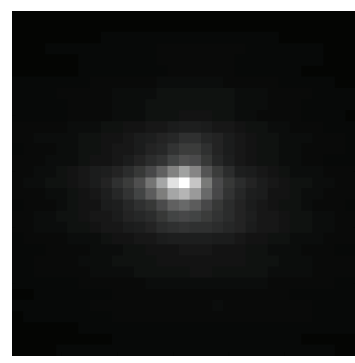

(c) $125 \times 125 \mu \mathrm{m}$ profile $(3 \mathrm{~K}$ - 100K photon counts).

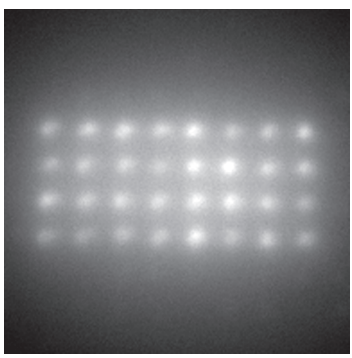

(d) CCD Image of $4 \times 8$ aligned laser spots.

Figure 5: Results from alignment experiments for single 5(a)-5(c) and multiple spots 5(d) using a scaled (min-max) linear grayscale.

\section{CONCLUSION}

An approach for automatic multi-laser spot to multi-pixel detector alignment has been proposed, providing a feasible solution to time-consuming optical alignment in multifocal microscopy experimentation. Although the technique can be ported to other 2D detector systems (e.g. EM-CCD), it is particularly suited to arrays with spatially separated (low fillfactor) pinhole detectors. The procedure for alignment is also independent of the SLM phase map generation and can therefore be used with any technique to produce laser spots using an SLM. Recent improvements in SLM technology, such as higher resolutions, smaller pixel sizes and increased switching speeds, will lead towards more efficient multiple confocal laser spot generation. The current detection system is capable of acquiring photon arrival time data for FCS and raw TCSPC values for FLIM, ${ }^{7}$ as well as providing real-time FLIM estimations, ${ }^{8}$ all of which would benefit significantly from the improved photon collection efficiency provided by multiple point confocal excitation and detection.

\section{ACKNOWLEDGMENTS}

Funding, equipment and support was supplied by; The University of Edinburgh, UK-EPSRC, FP6 MegaFrame Project, STMicroelectronics, Laboratoire Interdisciplinaire de Physique and The Royal Society of Edinburgh.

\section{REFERENCES}

[1] Gösch, M., Blom, H., Anderegg, S., Korn, K., Thyberg, P., Wells, M., Lasser, T., Rigler, R., Magnusson, A., and Hard, S., "Parallel dual-color fluorescence cross-correlation spectroscopy using diffractive optical elements," J. Biomed. Opt. 10 (2005).

[2] Blancquaert, Y., Gao, J., Derouard, J., and Delon, A., "Spatial fluorescence cross-correlation spectroscopy by means of a spatial light modulator," J. Biophotonics 1, 408-418 (2008).

[3] Colyer, R., Scalia, G., Rech, I., A.Gulinatti, Ghioi, M., Cova, S., Weiss, S., and Michalet, X., "High-throughput FCS using an LCOS spatial light modulator and an 8x1 SPAD array," Biomedical Optics Express 1(5), 1408-1431 (2010).

[4] Richardson, J., Walker, R., Grant, L., Stoppa, D., Borghetti, F., Charbon, E., Gersbach, M., and Henderson, R. K., "A 32x32 50ps Resolution 10 bit Time to Digital Converter Array in 130nm CMOS for Time Correlated Imaging," in [IEEE Custom Integrated Circuits Conference], (2009).

[5] Cojoc, D., Fabrizio, E. D., Businaro, L., and Cabrini, S., "Spherical-based approach to design diffractive optical elements," in [Proc. SPIE], 5227, 123-131 (2003).

[6] Richardson, J., Grant, L., and Henderson, R., "A Low Dark Count Single Photon Avalanche Diode Structure Compatible with Standard Nanometer Scale CMOS Technology," IEEE Photonics Technology Letters 21(14), 1020-1022 (2009).

[7] Giraud, G., Schulze, H., Li, D., Bachmann, T., Crain, J., Tyndall, D., Richardson, J., Walker, R., Stoppa, D., Charbon, E., Henderson, R., and Arlt, J., "Fluorescence lifetime biosensing with DNA microarrays and a CMOS-SPAD imager," Biomedical Optics Express 1(5), 1302-1308 (2010).

[8] Li, D., Arlt, J., Richardson, J., Walker, R., Buts, A., Stoppa, D., Charbon, E., and Henderson, R., "Real-time fluorescence lifetime imaging system with a $32 \times 320.13 \mu \mathrm{m}$ CMOS low dark-count single-photon avalanche diode array," Optics Express 18(10), 10257-10269 (2010). 